\title{
The transcriptional activator ZNF143 is essential for normal development in zebrafish
}

\author{
Kari M Halbig ${ }^{1}$, Arne C Lekven ${ }^{2}$ and Gary R Kunkel ${ }^{1^{*}}$
}

\begin{abstract}
Background: ZNF143 is a sequence-specific DNA-binding protein that stimulates transcription of both small RNA genes by RNA polymerase II or III, or protein-coding genes by RNA polymerase II, using separable activating domains. We describe phenotypic effects following knockdown of this protein in developing Danio rerio (zebrafish) embryos by injection of morpholino antisense oligonucleotides that target znf143 mRNA.

Results: The loss of function phenotype is pleiotropic and includes a broad array of abnormalities including defects in heart, blood, ear and midbrain hindbrain boundary. Defects are rescued by coinjection of synthetic mRNA encoding full-length ZNF143 protein, but not by protein lacking the amino-terminal activation domains. Accordingly, expression of several marker genes is affected following knockdown, including GATA-binding protein 1 (gata1), cardiac myosin light chain 2 ( $\mathrm{cm} / \mathrm{c} 2)$ and paired box gene 2a (pax2a). The zebrafish pax2a gene proximal promoter contains two binding sites for ZNF143, and reporter gene transcription driven by this promoter in transfected cells is activated by this protein.

Conclusions: Normal development of zebrafish embryos requires ZNF143. Furthermore, the pax2a gene is probably one example of many protein-coding gene targets of ZNF143 during zebrafish development.
\end{abstract}

\section{Background}

The vertebrate transcriptional activator protein, ZNF143 (also known as STAF for selenocysteine tRNA gene transcription activating factor, or SBF for SPH-binding factor) operates at a multitude of small RNA and protein-coding gene promoters [1-5]. Two separable activation domains within this protein stimulate transcription selectively at either small RNA or mRNA promoters [6]. Initially, attention was focused on the function of ZNF143 for small RNA gene transcription, especially for vertebrate snRNA and selenocysteine tRNA genes [7-9]. Then, several mRNA genes were identified whose proximal promoters contained SPH sites [10-15]. Possibly because of the highly degenerate and relatively long DNA-binding site recognized by ZNF143, it was not recognized for many years that approximately 2000 mammalian protein-coding genes contain SPH (SphI Postoctamer Homology [16]) elements, or STAF Binding Sites (SBS), in their promoters [5].

\footnotetext{
* Correspondence: g-kunkel@tamu.edu

'Department of Biochemistry and Biophysics, Texas A\&M University, College Station, TX 77843-2128, USA

Full list of author information is available at the end of the article
}

Little is known concerning the phenotypic role(s) of ZNF143 during cellular growth and animal development. A number of cell-cycle-associated gene promoters are regulated by ZNF143 [17-19]. Furthermore, ZNF143 is an important regulator of mammalian embryonic stem cell renewal $[20,21]$. At the molecular level, recent work has demonstrated that this activator protein interacts with the chromodomain-helicase-DNA binding protein 8 (CHD8), and implicates that human $U 6$ gene transcription is stimulated by ZNF143 through this interaction [22]. Many potential small RNA and protein-coding gene promoters are targeted, but which are most pivotal in vivo?

We used zebrafish embryos as a model system to investigate the role of ZNF143 during vertebrate development. Injection of translation-blocking morpholino oligonucleotides (MOs) resulted in a pleiotropic phenotype including axial defects as well as abnormalities in heart, blood, ear and midbrain hindbrain boundary (MHB). Coinjection of synthetic mRNA encoding zebrafish ZNF143 rescued MO-induced defects, and rescue was dependent on the amino-terminal region of the protein containing activation domains. Expression levels or patterns of the gata1, cmlc2, and pax $2 a$ genes were altered 
following MO knockdown of zebrafish ZNF143. The pax $2 a$ gene is likely to be a direct target for ZNF143 because this protein binds the promoter in vitro and specific mutations in SPH sites resulted in reduced transcription in transient transfection experiments.

\section{Results}

Identification of mRNA gene activation region in ZNF143 The zebrafish znf143 cDNA has been identified, and the predicted amino acid sequence contains a high degree of similarity with the human protein $(71 \%$ overall identity by our measurement) [23]. Furthermore, the zebrafish protein contains highly conserved regions that correspond to the previously identified DNA-binding domain (DBD), mRNA gene activation domain (15 aa repeats) and small RNA gene activation domain of the Xenopus and human proteins [23] (Figure 1A). To verify the mRNA gene activating potential of zebrafish ZNF143 and demarcate boundaries of this region, we fused fragments encoding zebrafish ZNF143 to the S. cerevisiae GAL4p DNA binding domain (amino acids 1-94), and performed transient transfection assays with such expression plasmids and a firefly luciferase reporter gene transcribed from a minimal promoter driven by GAL4 binding sites. Because transcriptional activating domains of the Xenopus protein were localized to the amino-terminal end previously [6], we investigated this region only. Amino acids 13-150 of zebrafish ZNF143 contains a potent mRNA gene activation region that functions in both human embryonic kidney (HEK293) cells and zebrafish ZF4 cells (Figure 1B, 1C). The region including only the four 15 aa repeats (amino acids 47-150) was approximately three-fold less active in both cell types. However, it is possible that this difference could be due to a lower expression level of this fragment (Figure 1B). Importantly, the region of zebrafish ZNF143 between the 15 aa repeats and the zinc finger domain (amino acids 151-228) demonstrated minimal mRNA promoter activation. Within this region has been identified a small RNA gene activating domain in the Xenopus protein [6].

\section{Knockdown of ZNF143 in zebrafish embryos}

To begin our studies with zebrafish embryos, we investigated the expression of $z$ nf143 RNA using whole-mount in situ hybridization. This RNA was expressed ubiquitously in the early stages that we examined, including 2-4 cell stage embryos and through gastrulation stages (results not shown). znf143 RNA must be inherited maternally, since it was detected prior to onset of zygotic transcription.

Next, we co-injected a pair of morpholino oligonucleotides (MOs) that targeted the translational start site and 5 ' untranslated region (5'UTR) of znf143 mRNA in order to reduce protein levels during embryonic development. Up to nearly $90 \%$ of injected embryos exhibited a range of defects at 48 hours post fertilization (hpf) (Figure 2). Microinjection of each individual MO resulted in the same range of phenotypes, but at a much lower efficiency (results not shown), supporting the assertion that the observed phenotypes are specific to ZNF143 knockdown. No mutant phenotypes were found when a control MO was injected at the same concentration (results not shown). For quantitation purposes, we sorted the morphant embryos into six classes that are primarily distinguished by increasing severity of axial and other defects. Class 1 embryos show a distinct kink in the tip of the tail (Figure 2B). Class 2 embryos have the same tail kink and also show disorganization in the somites (Figure 2C). Class 3 embryos have a greater degree of tissue disorganization as well as significant pericardial edema (Figure 2D). Class 4 embryos have the added phenotype of severe brain morphology defects, including absent or poorly formed midbrain-hindbrain boundary (MHB) (Figure $2 \mathrm{E})$. Class 5 embryos display the additional phenotype of a considerably shortened axis (Figure 2F). Class 6 morphants (not shown) are morphological monsters, lacking both head and tail structures. In addition to the described effects, we also noted reduced blood formation, rate of circulatory flow and abnormal ear development in significant proportions of morphant embryos falling in the more severe classes (classes 3-6).

Early developmental stages, through most of epiboly, proceeded normally for the ZNF143 morphant embryos, compared to embryos injected with the control MO. However, we detected a delay of approximately $30 \mathrm{~min}$ to reach bud stage. Furthermore, subsequent stages for ZNF143 morphants at 6-somite, 10-somite, 18-somite, and 22somite were delayed approximately one hour.

In order to verify the specificity of MO-knockdown, we co-injected synthetic mRNA encoding zebrafish ZNF143 with the MOs to determine whether protein synthesized from the exogenously supplied mRNA could rescue the phenotypes. We used mRNAs encoding either full length ZNF143 or a truncated variant lacking the transcription activation domains $(\Delta 2-225)$ in our rescue assays. Both mRNAs lack the normal 5'UTR sequence and encode a myc tag at the amino-terminus of the protein, such that the morpholinos will not block their expression. We first confirmed that the full-length myc-tagged protein is functional for transcriptional activation in transfected ZF4 cells (Figure 3A, compare lanes 2 and 3 with lanes 4 and 5). In addition, the $\Delta 2-225$ protein was defective for transcriptional activation in transfected ZF4 cells (Figure $3 \mathrm{~A}$, lanes 6 and 7). We detected nearly equivalent levels of expression of the myc-tagged full-length and $\Delta 2-225$ proteins in transfected HEK293 cells (Figure 3B). Thus, our constructs should be expressed at equivalent levels when expressed in embryos, even though for unknown reasons we have been unable to detect these proteins by 


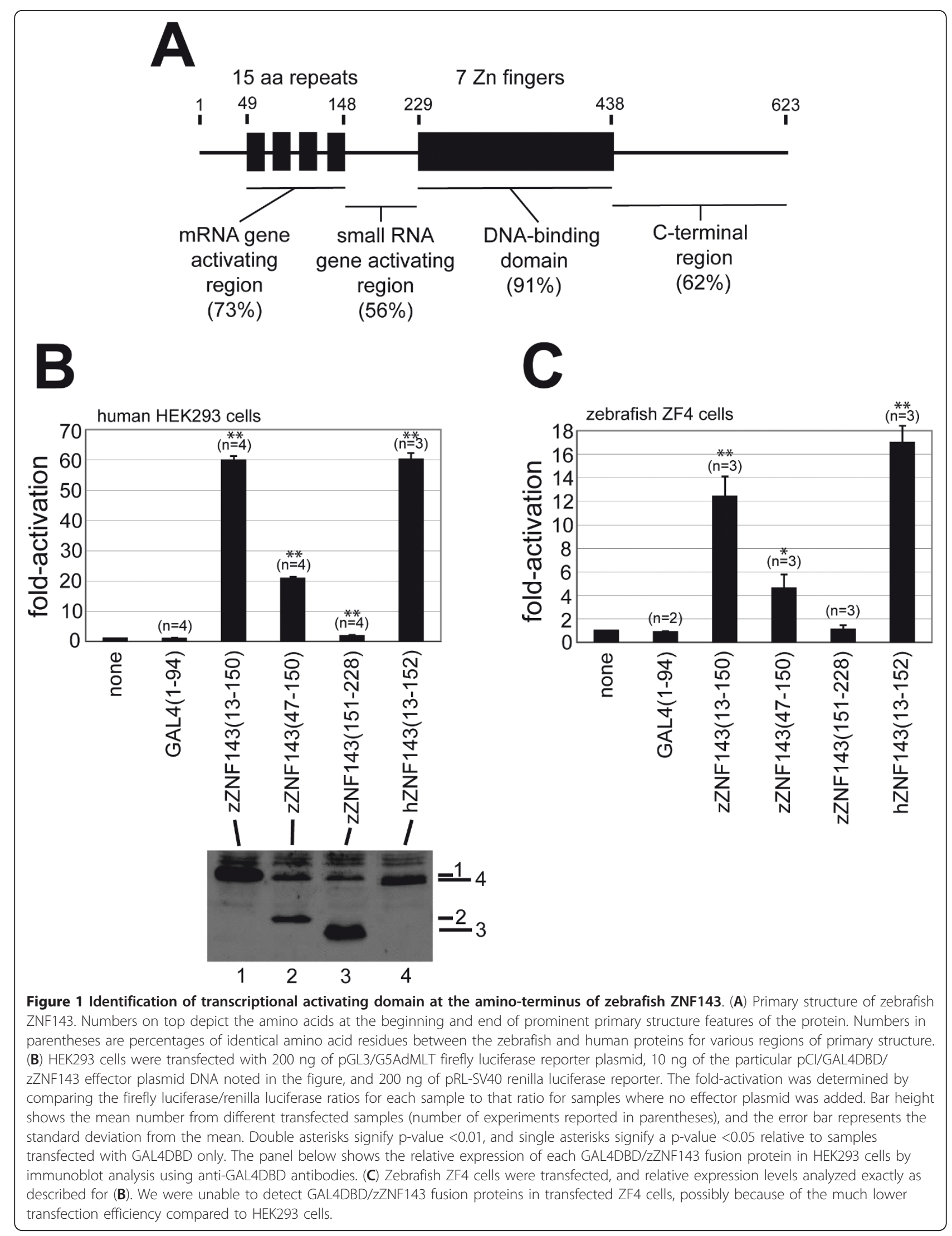




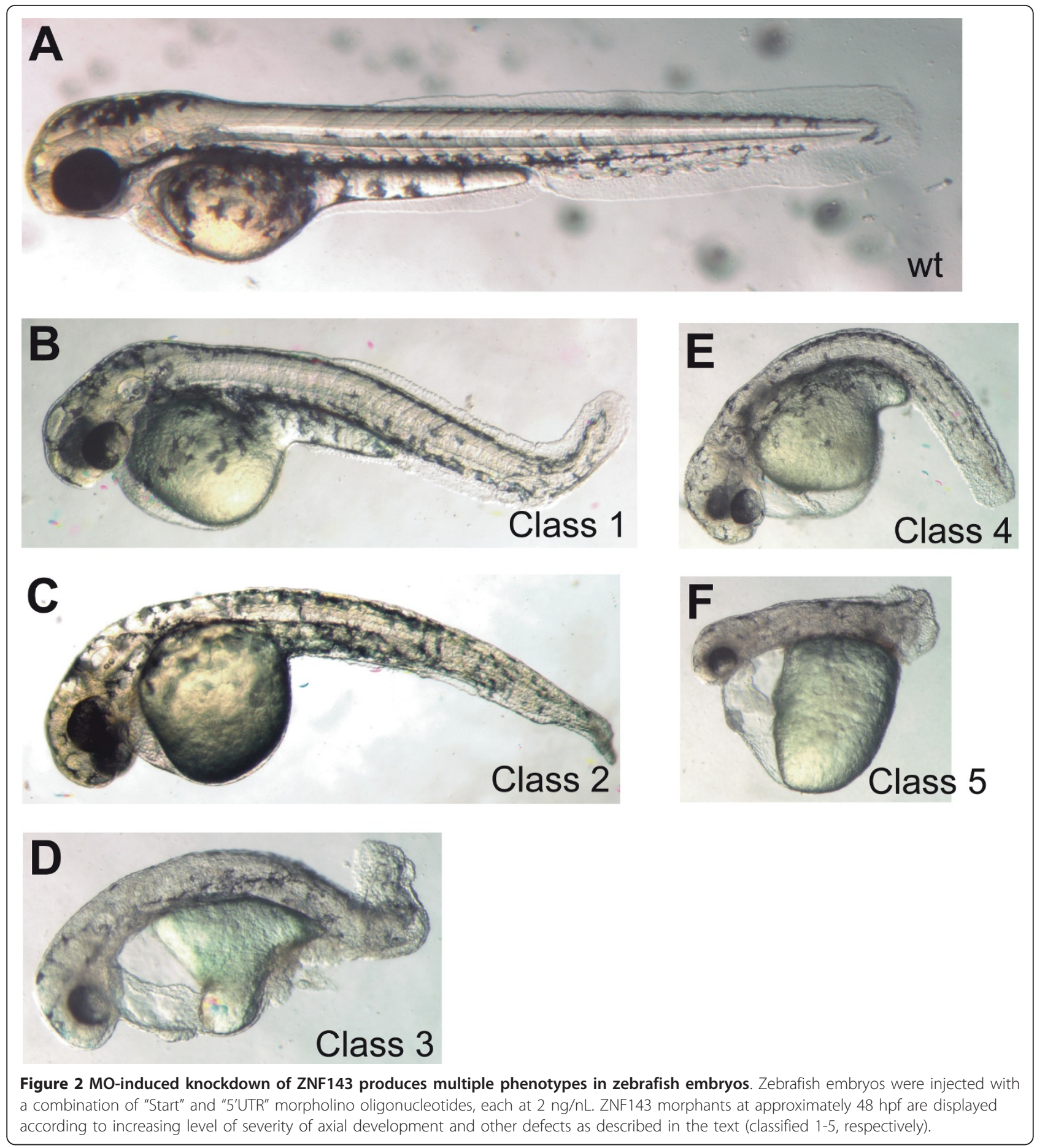

their myc-tags in injected embryos and in transfected ZF4 cells. Importantly, when co-injected with the MOs, full-length zebrafish ZNF143 rescued the general morphant phenotypes in approximately $70 \%$ of the injected embryos, and the remaining defects were primarily the least severe classes (Figure 3C, compare columns 1 and 2). The rescue was dependent on the presence of the amino-terminal region of zebrafish ZNF143 containing both activation domains, since co-injection of mRNA lacking codons 2-225 did not rescue the morphant phenotypes (Figure $3 \mathrm{C}$, column 3). These results show that the amino-terminal myc tag does not inactivate the function(s) of zebrafish ZNF143 in vivo, and the observed $\mathrm{MO}$-induced phenotypes are specific to the loss of 


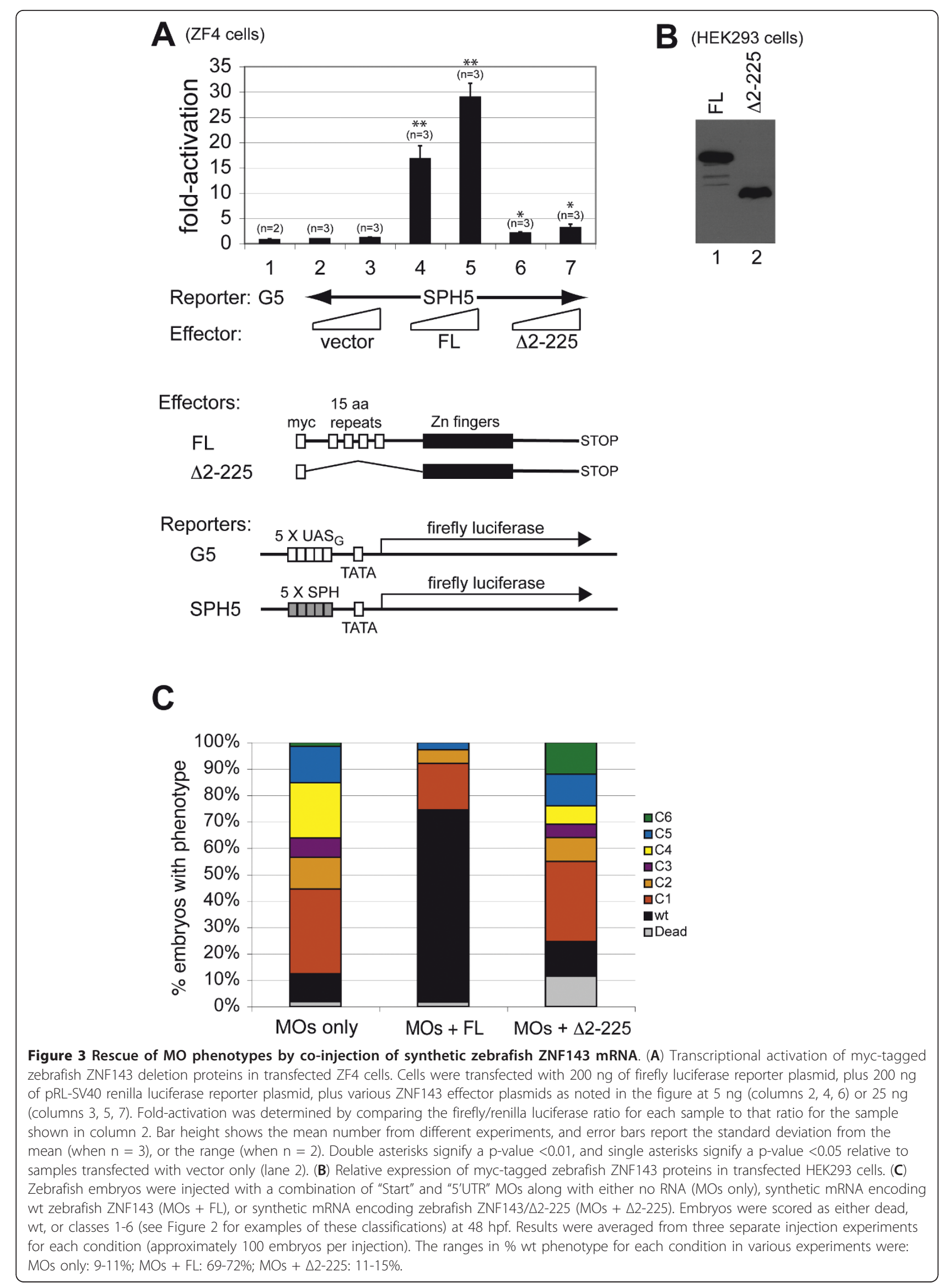


ZNF143 function and more specifically to ZNF143 transcriptional regulatory capability.

\section{pax2a expression is compromised after ZNF143 knockdown}

To determine whether the general morphological defects of ZNF143 morphants could be correlated with specific changes in gene expression, we performed whole mount in situ hybridization to detect developmental gene expression in morphant embryos at several stages. Using goosecoid $(g s c)$ and orthodenticle homolog 2 (otx2) probes to monitor prechordal plate mesoderm and neuroectoderm $[24,25]$, respectively, during gastrulation (shield stage and $75 \%$ epiboly for $g s c$, and $75 \%$ epiboly for otx 2 ), we detected no differences between ZNF143 morphants and embryos injected with a control MO (results not shown). In addition, hindbrain development of rhombomeres 3 and 5 appeared normal during early somitogenesis (6- and 10somite stages) using an early growth response 2a (egr $2 a$; or krox20) probe [26], although a delay in staining of rhombomere 5 was apparent in the 6-somite embryos (results not shown). This minor variation is consistent with the overall delay detected. Furthermore, we examined the expression of myoD, a marker for somitic muscle [27]. At all stages examined, we detected no discernable difference between wt and ZNF143 morphant embryos (5-6 somite, $\mathrm{n}=20 ; 16$ somite, $\mathrm{n}=13 ; 27 \mathrm{hpf}, \mathrm{n}=20$; data not shown). Next we analyzed the expression of gata1, a marker for hematopoietic progenitors [28], since blood development is compromised in ZNF143 morphants. At the 16-somite stage, gata1 labels populations of hematopoietic progenitors in the posterior mesoderm flanking the midline (Figure 4A). Consistent with our observation that blood specification is aberrant, ZNF143 morphants exhibited a significant reduction in gata1 expression at this stage (Figure 4B; 50\% penetrance, $\mathrm{n}=20$ ). At $24 \mathrm{hpf}$, gata1 is expressed in cells of the intermediate cell mass (ICM; Figure 4C), but expression continued to be reduced significantly in ZNF143 morphants (Figure 4D; 37\% penetrance, $n=19)$. Thus, the reduction in blood cells we observed in ZNF143 morphants correlates with a significant reduction in gata1 expression that labels hematopoietic progenitors.

We next analyzed expression of cardiac myosin light chain 2 ( $c m l c 2)$, a marker for cardiomyocytes, since heart development appeared disrupted after reduction of ZNF143. At $24 \mathrm{hpf}$, the heart comprises a tube that loops toward the left side of the embryo (Figure 4E). Heart progenitors formed normally in ZNF143 depleted embryos, but morphogenesis of the heart was severely compromised in $55 \%$ of MO-injected embryos (Figure 4F, 4G; $\mathrm{n}=38$ ). Phenotypic defects varied in this group from a failure to migrate to the left side of the embryo (Figure 4F), to a failure in tube morphogenesis (Figure
4G). These phenotypic defects in the ZNF143 morphants are not a result of prolonged delay of heart development, because embryos probed for $\mathrm{cmlc2}$ expression at $30 \mathrm{hpf}$ and $37 \mathrm{hpf}$ did not exhibit normal asymmetric looping (26/27 defective). At these later stages, $26 \%$ displayed cardia bifida (Figure 4H, 4I).

Because ZNF143 morphants displayed morphological abnormalities in the brain, including defects in the MHB, we analyzed the expression of pax $2 a$, a critical regulator of MHB formation. At the 16-somite stage, pax $2 a$ expression marks the optic stalks, mid-hindbrain domain, otic vesicles, pronephros and a small number of spinal cord neurons (Figure 4J). After reduction of ZNF143 function, pax $2 a$ expression was reduced in most expression domains of the majority of embryos $(68 \%, \mathrm{n}=19)$. Expression of pax $2 a$ in the MHB was reduced substantially in ZNF143 morphants, and to a lesser degree, expression in the otic vesicles, and pronephros (Figure 4K, 4L). In contrast, expression in the optic stalks did not appear to be significantly diminished compared to wt. Importantly, this phenotype was rescued by coinjection of wt ZNF143 mRNA ( $100 \%$ rescue, $\mathrm{n}=14$ ). At earlier stages (bud, 6somite, 10 -somite) pax $2 a$ expression was comparable between ZNF143 morphants and embryos injected with control MO. Furthermore, by $24 \mathrm{hpf}$, pax $2 a$ expression was not significantly different between wt and ZNF143 morphants, with the exception that pax $2 a$ expression in a group of hindbrain neurons was reduced or absent in the morphants (Figure 4M, 4N, arrows; $100 \%$ penetrance, $\mathrm{n}=$ 18). Thus, normal pax $2 a$ expression, particularly at the MHB, depends on ZNF143 function.

\section{The zebrafish pax2a gene promoter contains binding sites for ZNF143 and is controlled by this protein in transfected cells}

Since pax $2 a$ gene expression was affected after ZNF143 knockdown, we investigated whether this transcription factor might control zebrafish pax $2 a$ transcription. Visual inspection of the proximal promoter region sequence indicated three possible SPH motifs (Figure $5 \mathrm{~A})$. Radiolabeled probes were prepared that contained either the SPH1 element alone (-289/-131 fragment), or both SPH2 and SPH3 sites $(-154 /+16$ fragment). Both probes bound human ZNF143, showing a single complex that was competed with a specific human U6-1 $\mathrm{SPH}$ oligonucleotide, but not with an unrelated sequence containing the octamer (OCT) transcription control element (Figure 5B). Specific oligonucleotide competition for the pax2a DNA fragments was similar to that for the zebrafish U6 small nuclear RNA gene promoter used as a positive control for these experiments (Figure 5B, rightmost panel; [29]). Since only a single complex dependent on addition of a nonsaturating amount of ZNF143 was detected with the $-154 /+16$ 


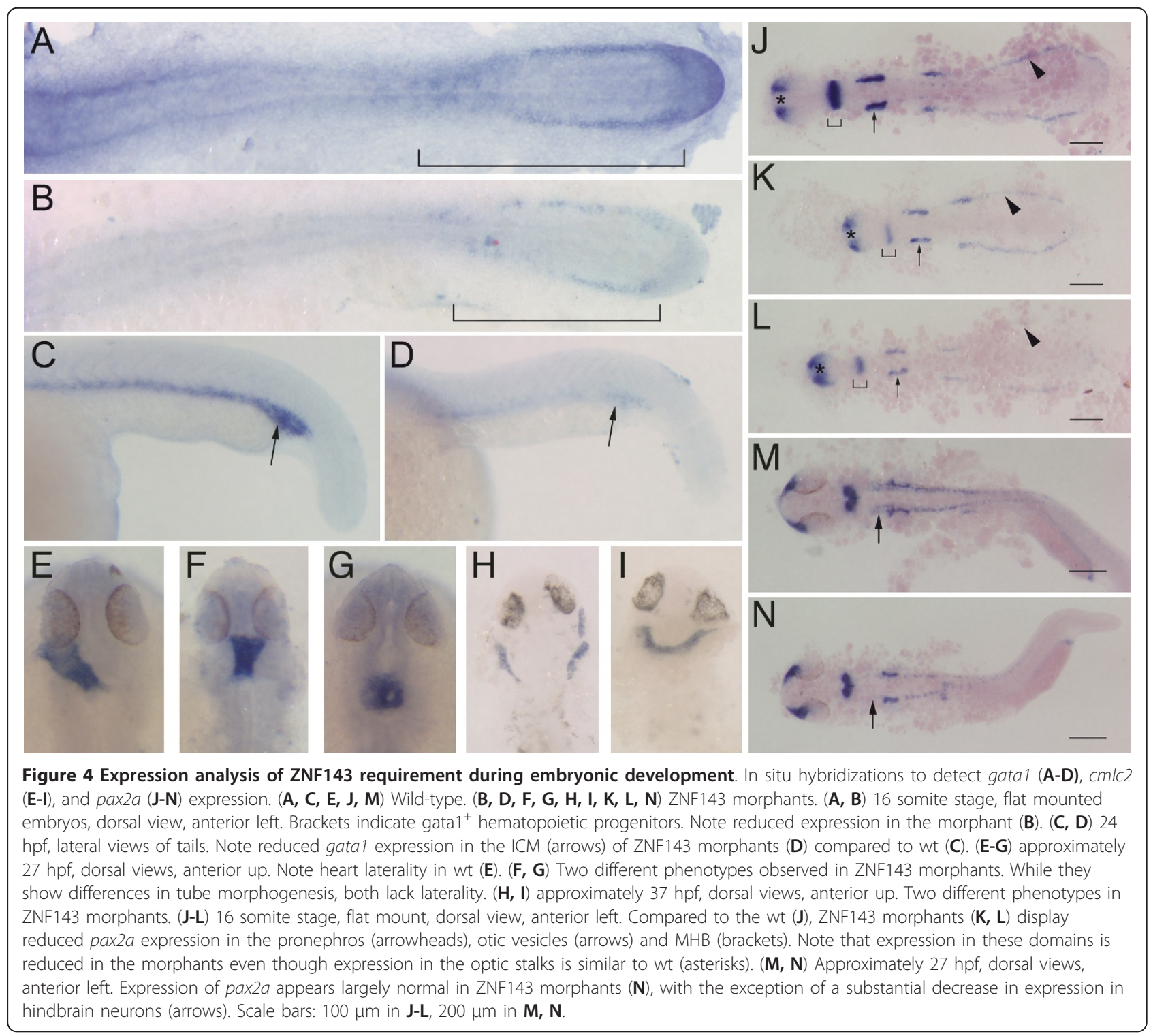

fragment, we suspected that only one of the two potential SPH elements was bound. DNaseI footprinting was used to determine which SPH element in the - 154/+16 fragment was bound by the protein. Results from footprinting experiments demonstrated binding of the human ZNF143 DNA-binding domain to the SPH2 element, while the SPH3 site was unoccupied at concentrations used for the experiment (Figure 5C. left panel). Therefore, two sites (SPH1 (likely) and SPH2) within the zebrafish pax $2 a$ promoter are bound by ZNF143 in vitro.

Next we investigated the transcriptional effect of the $\mathrm{SPH}$ sites in the zebrafish pax $2 a$ promoter in transient transfection experiments. Luciferase reporter plasmids containing either the wt pax2a promoter $(-289 /+16)$, single SPH1 or SPH2 mutants, or the double SPH1/2 mutant were added to zebrafish ZF4 cells. All mutant sites destroyed the highly conserved CCCA sequence of a SPH element (mutant sequences are shown in Methods). The efficacy of the SPH2MUT alteration was demonstrated by the obliteration of the DNaseI footprint in vitro (Figure 5C, lanes 6-9). The zebrafish pax $2 a$ proximal promoter $(-289 /+16)$ was quite active in transfected ZF4 cells since a typical experiment yielded approximately 7-fold higher normalized firefly luciferase level than from a simple TATA box only promoter (G5 reporter in Figure 3A). In transfected ZF4 cells, using a synthetic promoter containing multiple SPH elements, we found only low, basal activity at the same level as from a promoter lacking $\mathrm{SPH}$ sites (Figure 3A, compare columns 1 and 2). In human HEK293 cells the SPH5 promoter activity is approximately 20 -fold higher than 

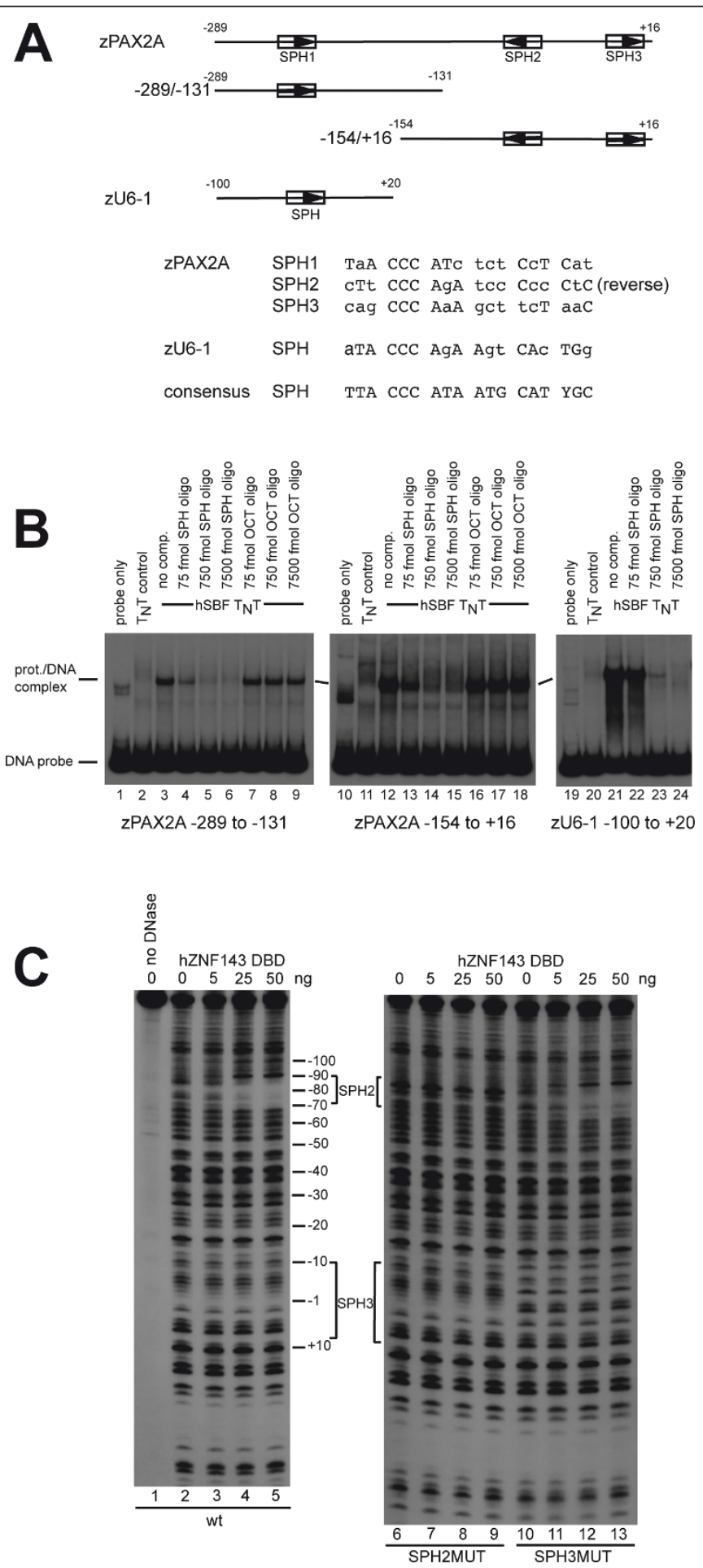

Figure 5 ZNF143 binds to the zebrafish pax2a promoter in vitro. (A) Map of zebrafish pax2a proximal promoter showing three potential SPH sites. The sequences of the potential SPH sites are compared to the zebrafish U6-1 element [29] and the consensus SPH sequence from [37]. Capital letters depict nucleotides matching the consensus. To facilitate binding assays two separate radiolabeled zebrafish pax2a probes were prepared by PCR as diagrammed. (B) Electrophoretic mobility shift assay. Approximately $3 \mathrm{fmol}$ of radiolabeled probes depicted in (A) were incubated with $3 \mu \mathrm{L}$ of human ZNF143-(88-638) expressed by in vitro transcription/translation (lanes 3-9; 12-18; 21-24) or with unprogrammed extract (lanes 2, 11, 20), and electrophoresed. In addition, in samples shown in lanes 4-6, 13-15, and 22-24, an unlabeled double-stranded oligonucleotide with the sequence including the human U6-1 SPH element was added with the labeled probe in amounts noted, or similarly, an unlabeled oligonucleotide containing the unrelated, OCT sequence was added to samples in lanes 7-9 and 16-18. (C) DNase I footprinting assay. Approximately $3 \mathrm{fmol}$ of singly end-labeled zebrafish pax2a probe from -154 to +16 were incubated with amounts of purified human ZNF143 DBD protein as indicated. The wt pax2a promoter was used for samples in lanes 1-5, whereas SPH2 or SPH3 mutant promoters were used for samples in lanes 6-9, or lanes 10-13, respectively. 
from the G5 promoter (unpublished results). Hence, unlike HEK293 cells, ZF4 cells do not contain sufficient amounts of active ZNF143 to stimulate potentially responsive promoters. Transcription from the wt pax $2 a$ promoter was increased almost two-fold by co-transfection with a ZNF143 expression plasmid (Figure 6). The SPH2MUT and doubly mutant promoters were not activated, whereas the relative stimulation of transcription from the SPH1MUT promoter was somewhat reduced (Figure 6, filled bars). Not surprisingly, transcription from the various SPHMUT pax $2 a$ promoters was not reduced significantly in the absence of exogenous
ZNF143 (unfilled bars in Figure 6). Hence the SPH1 and SPH2 elements are functional for transcriptional activity directed by the zebrafish $p a x 2 a$ gene promoter.

\section{Discussion}

In this study, we show that MO-induced knockdown of ZNF143 results in many phenotypic effects during zebrafish development. General defects in tail formation were obvious, but we also noticed abnormal heart, blood, ear and MHB development. The specificity of the MO-induced phenotypes was confirmed by experiments showing phenotypic rescue by the full-length protein

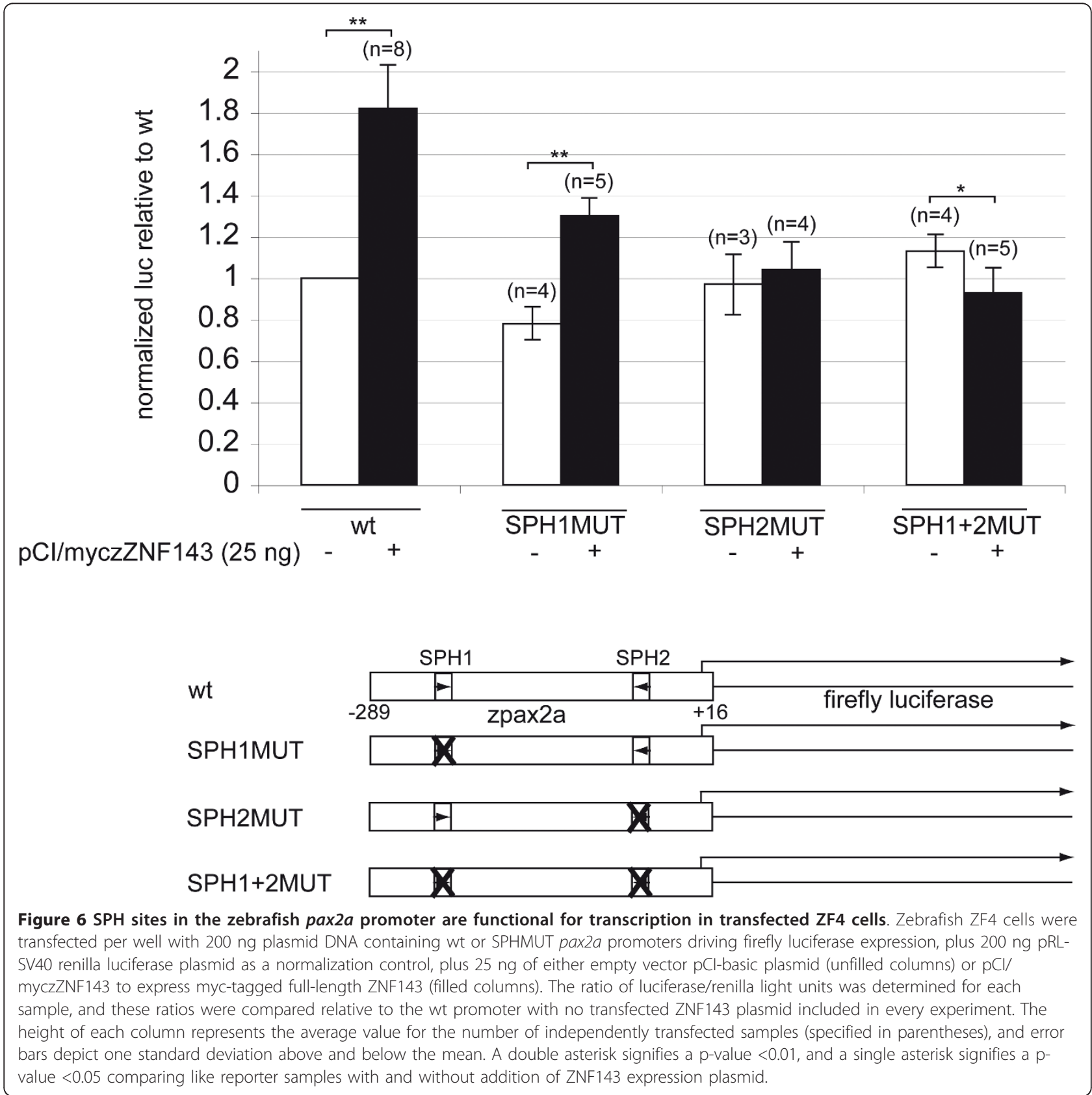


but not a truncated variant lacking transcriptional activation domains. In the morphants, expression of gatal (blood), cmlc2 (cardiac) and pax2a (MHB) were reduced or altered spatially. Furthermore, we found two binding sites for ZNF143 in the zebrafish pax2a gene proximal promoter, and disruption of these sites reduced transcription in transfected zebrafish cells. Therefore, one likely mRNA gene target for transcriptional control by this protein in vivo is pax $2 a$, although the number is probably much higher.

It is not surprising that a multitude of phenotypes appear after ZNF143 knockdown because many vertebrate small RNA genes contain SPH sites, and in mammals it has been hypothesized that approximately 2000 mRNA gene promoters contain SPH sites [5]. It seems likely that the gene encoding ZNF143 is essential for zebrafish viability. The number of promoters containing SPH elements in zebrafish is unknown. Previously, we have demonstrated an important positive role for the SPH site in zebrafish $U 6$ snRNA gene promoters [29], and have noticed putative SPH elements in many zebrafish small RNA gene promoters (unpublished results). Many fugu small RNA gene promoters contain SPH sites [23]. At present, we do not know which phenotypic effects or the relative severity of those effects caused by ZNF143 knockdown are the result of altered expression of small RNA genes or mRNA genes. Since knockdown was rescued by coinjection of synthetic mRNA encoding full-length ZNF143, but not by RNA lacking coding potential for $\mathrm{N}$-terminal activation domains (Figure 3), we hope to be able to distinguish differential effects of mRNA-activation vs. small RNA activation by this protein using rescue with synthetic RNA encoding deletions or mutations in those separable activation functions.

Since knockdown of ZNF143 caused relatively drastic phenotypic effects in zebrafish embryos, we were surprised that cultured zebrafish ZF4 cells contain undetectable levels of endogenous active protein in a transactivation assay (Figure 3A, compare reporter gene expression from G5 promoter vs. SPH5 promoter in columns 1-3). When these same reporter constructs were used in transfected human HEK293 cells the SPH5 promoter was transcribed at approximately 20-fold higher level due to endogenous ZNF143. Perhaps a small amount of ZNF143 in ZF4 cells is sufficient for their growth, and that amount is below a threshold detectable with relatively large amounts of reporter plasmid added in the transfection experiments. Indeed, mRNA encoding this protein in ZF4 cells was detected by RT-PCR (results not shown). Another possibility is that relative overexpression of ZNF143 in transfected cells suppresses another defect in the small RNA activation pathway in ZF4 cells. Nevertheless, the nonsaturating levels of active ZNF143 in ZF4 cells allowed us to use them as an assay for mRNA activation potential of tagged protein and protein lacking activation domains (Figure 3A), and we will use this assay in the future to examine activity of mutant proteins.

Our experiments demonstrating brain defects and, specifically, reduced pax $2 a$ expression upon ZNF143 knockdown induced us to inspect the pax2a proximal promoter for SPH sites. Indeed, ZNF143 binds to the pax2a promoter in vitro and activates it in an SPH-site dependent manner when cotransfected into ZF4 cells. Although we used existing human ZNF143 protein reagents in our laboratory for the binding studies, rather than the zebrafish protein, we expect that nucleotide specificity of the human and zebrafish proteins should be undistinguishable. The zinc finger DNA-binding domains are $91 \%$ identical, with no changes in the amino acid residues known to be most important for DNA recognition by this motif $[23,29,30]$. Although the enhancer(s) of the zebrafish $p a x 2 a$ gene promoter have been characterized partially [31], we are not aware of previous work to investigate elements within the proximal promoter. We expect that other elements in addition to the SPH1 and SPH2 sites constitute this promoter. Luciferase reporter gene activity of the SPH1+2 mutant promoter was approximately 7 -fold higher than that found with a simple TATA-containing promoter in our transient transfection assays (unpublished results). This promoter does not contain a readily identifiable TATA box, a characteristic that is true also of most previously identified SPH-containing mammalian mRNA gene promoters [5]. It will be interesting to dissect the pax $2 a$ promoter further in order to discern relative roles of SPH elements and other elements with respect to both basal transcription and enhancer-driven expression.

Because of the widespread abundance of SPH sites in mammalian mRNA gene promoters, we searched for them in other important developmental regulators in addition to pax2a. Although several SPH sites appeared to be candidates in the zebrafish gata1 proximal promoter, none bound ZNF143 in vitro (unpublished results). On the other hand, we have found a high-affinity SPH element in the zebrafish fibroblast growth factor receptor-1 ( $f g f r 1)$ and glycogen synthase kinase $3 \alpha$ promoters (unpublished results). Whether any of the aforementioned genes is controlled in a significant manner by ZNF143 will require further study. Furthermore, because of the large potential number of target promoters in mammals, it is highly likely that the transcription of many other developmental regulator genes is controlled by ZNF143.

\section{Conclusions}

Because knockdown of the transcriptional activator ZNF143 by injection of translational-blocking MOs causes many significant phenotypic effects in zebrafish embryos, we conclude that this protein is essential for 
normal development. Phenotypic effects are rescued by coinjection of full-length znf143 mRNA, but not by mRNA lacking coding capacity for the $\mathrm{N}$-terminal region containing activation domains. Hence, the MOmediated knockdown is specific, and ZNF143 function in vivo requires the $\mathrm{N}$-terminal region. Furthermore, the pax2a gene promoter is at least one likely target of ZNF143 during zebrafish development.

\section{Methods}

\section{Plasmid constructions}

A cDNA containing the zebrafish $z n f 143$ gene was obtained from Open Biosystems. We named this plasmid pME18S-FL3/zZNF143. The open reading frame (ORF) contained within this insert was lacking a full-length gene and contained a reading frame error in the coding region near the amino-terminus of the encoded protein. Hence, in order to construct a full-length ORF situatated behind a T7 promoter, three DNA fragments were ligated as follows. The "correct" amino-terminal region fragment was prepared by reverse transcriptase polymerase chain reaction (RT-PCR) using total RNA from zebrafish ZF4 cells and ligated into a pGEM-T vector (Promega). This fragment was excised using $K p n \mathrm{I}$ and $P v u \mathrm{II}$ and purified by agarose gel electrophoresis. The main body of the znf143 ORF was excised from pME18S-FL3/zZNF143 using PvuII and $\mathrm{XhoI}$ and purified by agarose gel electrophoresis. The third DNA fragment was the pBlueScript SK vector (Stratagene) opened at $K p n I$ and $\mathrm{XhoI}$ sites, and purified by chromatography on Sepharose CL-4B. The three-fragment ligation reaction was used to transform $E$. coli XL1-Blue (Stratagene) competent cells. The resulting plasmid was named pBS/zZNF143. A single myc tag was inserted at the amino-terminus of the encoded ZNF143 protein using the QuickChange site-directed mutagenesis protocol. The subsequent $\Delta 2-225$ deletion within the zebrafish $z$ nf 143 ORF started with the parent plasmid pBS/myczZNF143 and was constructed using the QuickChange protocol in which the oligonucleotides base-paired across the deletion endpoints and looped out the template DNA to be deleted. Plasmid minipreps were prepared using a Qiagen miniprep kit, and such preparations were suitable for mRNA synthesis in vitro. Zebrafish ZNF143 expression plasmids used for transient transfection experiments were constructed by amplifying inserts from pBS-based plasmids using PfuUltraHF polymerase (Stratagene), restriction with $\mathrm{XbaI}$ and SalI, gel-purification, and ligation into like sites in the pCI-neo vector (Promega). GAL4DBD - zebrafish ZNF143 fusion expression plasmids were constructed in the pCIneo vector (Promega), and contained inserts encoding the amino-terminal 94 aa of S. cerevisiae Gal4p [11] fused inframe to zebrafish ZNF143 fragments amplified by PCR. Luciferase reporter plasmids used for transient transfection experiments were based upon the pGL3-basic vector
(Promega) and included the adenovirus major late basal promoter (AdMLT). The pGL3/G5AdMLT (“G5") reporter contained five copies of the $S$. cerevisiae upstream activating sequence galactose $\left(\mathrm{UAS}_{\mathrm{G}}\right)$ element ligated upstream of the TATA box, whereas the "SPH5" reporter contained five copies of the human U6-1 gene SPH element in the same position. The zebrafish pax2a gene promoter $(-289$ to +16$)$ was amplified from genomic DNA by PCR and ligated into the pGEM-T vector (Promega). The location of the transcriptional start site $(+1)$ is according to the 5'-most expressed sequence tag (EST) in the University of California, Santa Cruz zebrafish genome assembly (July 2007 assembly, chromosome 13, position 29,268,562). SPHMUT plasmids containing clustered point mutations within SPH sites of the pax $2 a$ promoter were prepared by the QuickChange protocol. The nucleotide changes introduced into SPHMUT sites were (mutant nucleotides in lower case letters): SPHMUT1: 5'-TAAgatcTCTCTCCTCAT-3'; SPH2MUT: 5'-CTagatctATCC CCCCTC-3' (bottom strand); SPH3MUT: 5'-CAagatctAGCTTCTAAC-3' (top strand). DNA fragments containing wt and mutant pax $2 a$ promoters were amplified by PCR using pGEM-based templates, restricted with SacI and NheI, and ligated into the same sites of the pGL3basic vector. Inserts of all plasmids were sequenced completely to verify deletions, in-frame ligations, and ensure that no other mutations were added to the $z n f 143$ ORF or pax $2 a$ promoter. DNAs used for transient transfection experiments were purified using the Qiagen plasmid maxi kit, and concentrations were determined by absorbance at $260 \mathrm{~nm}$.

\section{Cell culture, transfection, and reporter gene assays}

Human HEK293 cells were purchased from ATCC (CRL1573) and cultured in Dulbecco's Modified Eagle Medium (DMEM), containing high glucose (Gibco 11995), penicillin-streptomycin, and 10\% bovine growth serum (Hyclone). Experiments with HEK293 cells were performed under BSL-2 guidelines following approval by the Texas A\&M University Institutional Biosafety Committee. Zebrafish ZF4 cells [32] were obtained from ATCC (CRL2050) and grown in DMEM/F12 medium (Gibco 11320), penicillin-streptomycin, and $10 \%$ bovine growth serum. Cells were transfected in 6-well dishes using Lipofectamine 2000 (Invitrogen) following the manufacturer's protocol with amounts of DNA described in the figure legends. Cells were co-transfected with pRL-SV40 (Promega), a renilla luciferase reporter, for normalization. $48 \mathrm{~h}$ post-transfection, cell extracts were prepared, and firefly and renilla luciferase activities were measured with a Sirius tube luminometer (Berthold) using reagents and protocols from the Dual-Luciferase Reporter Assay System (Promega). The number of independently transfected samples for each condition is shown in parentheses above the bars 
in the graphs. Statistical significance (p-values) comparing sets of different experiments was calculated using the Student's T test in Microsoft Excel.

\section{Electrophoretic mobility shift assay (EMSA) and DNasel footprinting}

Two radiolabeled pax $2 a$ gene proximal promoter probes used for protein-DNA binding assays were prepared by PCR using either ${ }^{32} \mathrm{P}$-end-labeled CZPAX2A-131 primer (5'-CAACACTTTGTGATTCGCCAACGC-3'), and unlabeled ZPAX2A-289 primer (5'-TGGTACCGCTTCCTTT CCACTTGT-3'), or ${ }^{32} \mathrm{P}$ end-labeled CZPAX2A+16 primer (5'ATGTGCCTGTTAGAAGCTTTGGGC-3') and unlabeled ZPAX2A-154 primer (5'-GCGTTGGCGAATCACAAAGTGTTG-3'), and pGEM plasmid DNA with wt or SPHMUT pax $2 a$ promoter inserts. The zebrafish $U 6-1$ probe was prepared as described [29]. Protein for EMSA experiments was prepared by in vitro transcription/ translation of human znf143 as described previously [9]. DNA-protein complex formation, competition with oligonucleotides, and electrophoresis on non-denaturing gels were performed as before [33]. Sequences of the competitor oligonucleotides can be found in [2] where human U6$1 \mathrm{SPH}$ is called NONOCT(long) and human U6-1 OCT is called OCTCON. DNaseI footprinting assays followed the same protocol as described previously [33], using purified human ZNF143 zinc finger DNA-binding domain (DBD) (amino acids 236-444) expressed from a pET5a vector in E. coli [29]. The positions of the putative SPH elements were deduced by electrophoresis of $\mathrm{T}$ and $\mathrm{C}$ dideoxy sequencing reactions on the same gels.

\section{Zebrafish husbandry and embryo microinjection}

AB strain zebrafish were maintained using standard methods [34], and with protocols approved by the Texas A\&M Animal Care and Use Committee (AUP \#2007-90 and 2010-90). MOs and synthetic mRNAs were injected at the one- to two-cell stage. Two MOs were designed to block the translation of zebrafish $z n f 143$, dissolved in $1 \mathrm{X}$ Danieau buffer, and were coinjected at a concentration of $2 \mathrm{ng} / \mathrm{nL}$, each. The "Start" MO, ordered from Open Biosystems and Gene Tools, LLC, had the sequence, 5'-ATTCACCTGGGCTAACAGCATGATC-3', and the " 5 'UTR" MO, ordered from Gene Tools, LLC, had the sequence, 5'CAACAATCCCTTCGTTCGACCACCA-3'. The control MO (5'-CCTCTTACCTCAGTTACAATTTATA-3') was purchased from Gene Tools, LLC. For rescue coinjection experiments, capped and polyadenylated mRNAs were synthesized from pBS/myczZNF143 or the $\Delta 2-225$ deletion variant template using the mMESSAGE mMACHINE T7 Ultra kit (Ambion). Synthetic mRNA concentrations were determined using a NanoDrop spectrophotometer. Approximately $3 \mathrm{~nL}$ of RNAs were injected at a concentration of $30 \mathrm{pg} / \mathrm{nL}$, an amount that did not cause any toxicity or phenotype when injected without MOs. Phenotypes were classified for quantitation in Figure $3 \mathrm{C}$ at 48 hpf.

\section{Immunoblots}

Nuclear extracts from HEK293 cells were prepared using the NE-PER kit (Pierce/Thermo Scientific). After electrophoresis on standard SDS denaturing gels, proteins were transferred to nitrocellulose, and probed with rabbit anti-yeast GAL4DBD (Upstate/Millipore), or mouse monoclonal anti-myc (Upstate/Millipore, clone 4A6).

\section{Whole-mount in situ hybridization}

Whole-mount in situ hybridizations with embryos were performed according to standard methods [26]. Probes used were $c m l c 2$ [35], pax2a [36], gata1 [28], myoD [27], gsc [24], otx2 [25], and egr2a (or krox20) [26]. The numbers of independently injected embryos scored for various phenotypes are noted in the text.

\section{Acknowledgements}

This work was supported by funds from the Department of Biochemistry and Biophysics (TAMU). ACL is a Research Scholar of the American Cancer Society (RSG-06-150-01-DDC). We thank members of the Lekven laboratory (Cathryn Kelton, Anand Narayanan, Kevin Baker, and Amy Whitener) for assistance with fish husbandry, in situ protocols and for supplying probes for myoD, gata1, cmlc2, pax2a, gsc, otx2, and krox20. The Riley laboratory (TAMU) also supplied the probe for paxza for some experiments.

\section{Author details}

${ }^{1}$ Department of Biochemistry and Biophysics, Texas A\&M University, College Station, TX 77843-2128, USA. ${ }^{2}$ Department of Biology, Texas A\&M University, College Station, TX 77843-3258, USA.

\section{Authors' contributions}

$\mathrm{KMH}$ designed and carried out experiments shown in Figures 2, 3C, and 4. $\mathrm{ACL}$ participated in the design of the study, the microscopy and the preparation of the manuscript. GRK performed experiments shown in Figures 1, 2A,B, 5, and 6, conceived the project, coordinated the work, and drafted the manuscript. All authors read and approved the final manuscript.

Received: 23 September 2011 Accepted: 23 January 2012 Published: 23 January 2012

\section{References}

1. Zamrod Z, Stumph WE: U4B snRNA gene enhancer activity requires functional octamer and SPH motifs. Nucleic Acids Res 1990, 18:7323-7330.

2. Kunkel GR, Cheung TC, Miyake JH, Urso O, McNamara-Schroeder KJ, Stumph WE: Identification of a SPH element in the distal region of a human U6 small nuclear RNA gene promoter and characterization of the SPH binding factor in HeLa cell extracts. Gene Expr 1996, 6:59-72

3. Schaub M, Myslinski E, Schuster C, Krol A, Carbon P: Staf, a promiscuous activator for enhanced transcription by RNA polymerases II and III. EMBO J 1997, 16:173-181.

4. Myslinski E, Krol A, Carbon P: ZNF76 and ZNF143 Are Two Human Homologs of the Transcriptional Activator Staf. J Biol Chem 1998, 273:21998-22006.

5. Myslinski E, Gerard MA, Krol A, Carbon P: A Genome Scale Location Analysis of Human Staf/ZNF143-binding Sites Suggests a Widespread Role for Human Staf/ZNF143 in Mammalian Promoters. J Biol Chem 2006, 281:39953-39962. 
6. Schuster C, Krol A, Carbon P: Two Distinct Domains in Staf To Selectively Activate Small Nuclear RNA-Type and mRNA Promoters. Mol Cell Biol $1998,18: 2650-2658$.

7. Roebuck KA, Szeto DP, Green KP, Fan QN, Stumph WE: Octamer and SPH Motifs in the U1 Enhancer Cooperate To Activate U1 RNA Gene Expression. Mol Cell Biol 1990, 10:341-352.

8. Schuster C, Myslinski E, Krol A, Carbon P: Staf, a novel zinc finger protein that activates the RNA polymerase III promoter of the selenocysteine tRNA gene. EMBO J 1995, 14:3777-3787.

9. Rincon JC, Engler SK, Hargrove BW, Kunkel GR: Molecular cloning of a CDNA encoding human SPH-binding factor, a conserved protein that binds to the enhancer-like region of the U6 small nuclear RNA gene promoter. Nucleic Acids Res 1998, 26:4846-4852.

10. Kubota H, Yokota S, Yanagi H, Yura T: Transcriptional Regulation of the Mouse Cytosolic Chaperonin Subunit Gene Ccta/t-Complex Polypeptide 1 by Selenocysteine tRNA Gene Transcription Activating Factor Family Zinc Finger Proteins. J Biol Chem 2000, 275:28641-28648.

11. Mach CM, Hargrove BW, Kunkel GR: The Small RNA Gene Activator Protein, Sphl Postoctamer Homology-binding Factor/Selenocysteine tRNA Gene Transcription Activating Factor, Stimulates Transcription of the Human Interferon Regulatory Factor-3 Gene. J Biol Chem 2002, 277:4853-4858

12. Saur D, Seidler B, Paehge $H$, Schusdziarra V, Allescher HD: Complex Regulation of Human Neuronal Nitric-oxide Synthase Exon 1c Gene Transcription. J Biol Chem 2002, 277:25798-25814.

13. Barski OA, Pupusha VZ, Kunkel GR, Gabbay KH: Regulation of aldehyde reductase expression by STAF and CHOP. Genomics 2004, 83:119-129.

14. Di Leva F, Ferrante MI, Demarchi F, Caravelli A, Matarazzo MR, Giacca M, D'Urso M, D'Esposito M, Franze A: Human Synaptobrevin-like 1 Gene Basal Transcription Is Regulated through the Interaction of Selenocysteine tRNA Gene Transcription Activating Factor-Zinc Finger 143 Factors with Evolutionary Conserved Cis-elements. J Biol Chem 2004, 279:7734-7739.

15. Grossman CE, Qian Y, Banki K, Perl A: ZNF143 Mediates Basal and Tissuespecific Expression of Human Transaldolase. J Biol Chem 2004, 279:12190-12205.

16. Roebuck KA, Walker RJ, Stumph WE: Multiple Functional Motifs in the Chicken U1 RNA Gene Enhancer. Mol Cell Biol 1987, 7:4185-4193.

17. Myslinski E, Gerard MA, Krol A, Carbon P: Transcription of the human cell cycle regulated BUB1B gene requires hStaf/ZNF143. Nucleic Acids Res 2007, 35:3453-3464.

18. Izumi $H$, Wakasugi $T$, Shimajiri $S$, Tanimoto A, Sasaguri $Y$, Kashiwagi $E$, Yasuniwa Y, Akiyama M, Han B, Wu Y, et al: Role of ZNF143 in tumor growth through transcriptional regulation of DNA replication and cellcycle-associated genes. Cancer Sci 2010, 101:2538-2545.

19. Hernandez-Negrete I, Sala-Newby GB, Perl A, Kunkel GR, Newby AC, Bond M: Adhesion-Dependent skp2 Transcription Requires Selenocysteine tRNA Gene Transcription Activating Factor (STAF). Biochem J 2011, 436:133-143.

20. Chen X, Fang F, Liou YC, Ng HH: Zfp143 Regulates Nanog Through Modulation of Oct4 Binding. Stem Cells 2008, 26:2759-2767.

21. Chia NY, Chan YS, Feng B, Lu X, Orlov YL, Moreau D, Kumar P, Yang L, Jiang J, Lau MS, et al: A genome-wide RNAi screen reveals determinants of human embryonic stem cell identity. Nature 2010, 468:316-320.

22. Yuan CC, Zhao X, Florens L, Swanson SK, Washburn MP, Hernandez N: CHD8 Associates with Human Staf and Contributes to Efficient U6 RNA Polymerase III Transcription. Mol Cell Biol 2007, 27:8729-8738

23. Myslinski E, Krol A, Carbon P: Characterization of snRNA and snRNA-type genes in the pufferfish Fugu rubripes. Gene 2004, 330:149-158.

24. Schulte-Merker S, Hammerschmidt M, Beuchle D, Cho KW, De Robertis EM, Nusslein-Volhard C: Expression of zebrafish goosecoid and no tail gene products in wild-type and mutant no tail embryos. Dev 1994, 120:843-852.

25. Li Y, Allende ML, Finkelstein R, Weinberg ES: Expression of two zebrafish orthodenticle-related genes in the embryonic brain. Mech Dev 1994 48:229-244.

26. Oxtoby $\mathrm{E}$, Jowett $\mathrm{T}$ : Cloning of the zebrafish krox-20 gene (krx-20) and its expression during hindbrain development. Nucleic Acids Res 1993 21:1087-1095.

27. Weinberg ES, Allende ML, Kelly CS, Abdelhamid A, Marakami T, Andermann P, Doerrre OG, Grunwald DJ, Riggleman B: Developmental regulation of zebrafish MyoD in wild-type, no tail and spadetail embryos. Dev 1996, 122:271-280.

28. Detrich HW, Kieran MW, Chan FY, Barone LM, Yee K, Rundstadler JA, Pratt S, Ransom D, Zon LI: Intraembryonic hematopoietic cell migration during vertebrate development. Proc Natl Acad Sci USA 1995, 92:10713-10717.

29. Halbig KM, Lekven AC, Kunkel GR: Zebrafish U6 small nuclear RNA gene promoters contain a SPH element in an unusual location. Gene 2008, 421:89-94

30. Pavletich NP, Pabo CO: Zinc finger-DNA recognition: Crystal structure of a Zif268- DNA complex at 2.1 Å. Science 1991, 252:809-817.

31. Picker A, Scholpp S, Bohli H, Takeda H, Brand M: A novel positive transcriptional feedback loop in midbrain-hindbrain boundary development is revealed through analysis of the zebrafish pax2.1 promoter in transgenic lines. Dev 2002, 129:3227-3239.

32. Driever W, Rangini Z: Characterization of a Cell Line Derived from Zebrafish (Brachydanio rerio) Embryos. In Vitro Cell Dev Biol 1993, 29A:749-754.

33. Danzeiser DA, Urso O, Kunkel GR: Functional characterization of elements in a human U6 small nuclear RNA gene distal control region. Mol Cell Biol 1993, 13:4670-4678.

34. Westerfield M: The Zebrafish Book. A Guide for the Laboratory Use of Zebrafish (Danio rerio). 4 edition. Eugene: The University of Oregon Press; 2000.

35. Yelon D, Horne SA, Stainier DY: Restricted expression of cardiac myosin genes reveals regulated aspects of heart tube assembly in zebrafish. Dev Biol 1999, 214:23-37.

36. Krauss $\mathrm{S}$, Johansen $\mathrm{T}$, Korzh V, Fjose A: Expression pattern of zebrafish pax genes suggests a role in early brain regionalization. Nature 1991, 353:267-270

37. Schaub M, Krol A, Carbon P: Flexible Zinc Finger Requirement for Binding of the Transcriptional Activator Staf to U6 Small Nuclear RNA and tRNA ${ }^{\text {Sec }}$ Promoters. J Biol Chem 1999, 274.24241-24249.

doi:10.1186/1471-2199-13-3

Cite this article as: Halbig et al:: The transcriptional activator ZNF143 is essential for normal development in zebrafish. BMC Molecular Biology 2012 13:3.

\section{Submit your next manuscript to BioMed Central and take full advantage of:}

- Convenient online submission

- Thorough peer review

- No space constraints or color figure charges

- Immediate publication on acceptance

- Inclusion in PubMed, CAS, Scopus and Google Scholar

- Research which is freely available for redistribution 